\title{
Estudio exploratorio: relación entre nivel de pobreza y exposiciones agudas a sustancias potencialmente tóxicas en Chile
}

\author{
Exploratory study: Association between the poverty level and acute exposures \\ to potentially toxic substances in Chile
}

\author{
Patricio Andrés Medel-Jara 1,2,3, Jaime Cerda Lorca ${ }^{5}$, E.M. Alberto López-Nuñez.1, Lorena Silva. ${ }^{1}$, \\ Dra. Sandra Solari Gajardo 1,2,4, Juan Carlos Ríos Bustamante ${ }^{1,2,4}$
}

\begin{abstract}
Resumen
Objetivo: explorar la siguiente pregunta de investigación: ¿De qué manera el nivel socioeconómico de una persona, específicamente el nivel de pobreza se asocia a las características de las exposiciones agudas a sustancias con potencial tóxico en Chile? Métodos: estudio epidemiológico, de diseño transversal y semi-ecológico. Se recopiló información proveniente de una muestra de casos atendidos por el Centro de información toxicológica y de medicamentos de la Pontificia Universidad Católica de Chile (CITUC). La variable independiente corresponde al índice de pobreza comunal de la Encuesta de Caracterización Socioeconómica Nacional (CASEN). Las variables dependientes fueron las características de las exposiciones registradas por el centro toxicológico de acuerdo con el nivel de pobreza de los casos observados. Se analizó el fenómeno a través de tablas cruzadas, cálculo de Odds Ratio con análisis estratificado. Resultados: se observan diferencias significativas en las circunstancias de las exposiciones: las personas que viven en comunas con mayor porcentaje de pobreza muestran más exposiciones intencionales versus aquellas con menor porcentaje de pobreza. Otro hallazgo significativo es el uso del sistema de salud en casos de exposiciones dependiente de la situación socioeconómica. Para las otras variables no se encuentran diferencias significativas entre los grupos comparados. Conclusiones: se observa una relación entre el nivel de pobreza y las exposiciones a sustancias potencialmente tóxicas. Los resultados abren una línea investigativa y nuevas interrogantes que podrían generar medidas de control de las intoxicaciones en Chile.
\end{abstract}

Palabras clave: toxicología; exposición a compuestos químicos; envenenamiento; determinantes sociales de la salud

\begin{abstract}
Objective: explore the following research question: How is a person's socio-economic level, the poverty, associated with the characteristics of acute exposures to substances with toxic potential in Chile?. Methods: epidemiological, cross-sectional and semi-ecological study. Information collected from a sample of cases attended by the Centre for Toxicological and Drug Information of the Pontifical Catholic University of Chile (CITUC). The independent variable corresponds to the communal poverty index of the National Socioeconomic Characterization Survey. Dependent variables were the characteristics of the exposures recorded by the toxicological center according to the poverty level of the cases observed. The phenomenon is analyzed through cross-tables, calculating Odds Ratios with stratified analysis. Results: significant differences observed in the circumstances of exposures: people living in communes with higher poverty rates show more intentional exposures versus those with lower poverty rates. Another significant finding is the use of the health system in cases of exposures, depending on the socio-economic situation. For the other variables, there are no significant differences between the compared groups. Conclusions: there is a link between the poverty level and acute exposures to potentially toxic substances. The results open a research line and new questions that could generate measures to control poisonings in Chile.
\end{abstract}

Keywords: poisoning; social determinants of health; poverty

Fecha de envío: 19 de agosto de 2019 - Fecha de aceptación: 26 de diciembre de 2019

(1) Centro de Información Toxicológica y de Medicamentos, Pontificia Universidad Católica de Chile.

(2) Unidad docente de Farmacología y Toxicología, Facultad de Medicina, Pontificia Universidad Católica de Chile.

(3) Departamento de Salud del Adulto y Senescente, Escuela de Enfermería, Pontificia Universidad Católica de Chile.

(4) Departamento de Laboratorios Clínicos, Facultad de Medicina, Pontificia Universidad Católica de Chile.

(5) Departamento de Salud Pública, Facultad de Medicina, Pontificia Universidad Católica de Chile.

Autor de correspondencia: Juan Carlos Ríos jriosb@uc.cl

ARS MEDICA Revista de Ciencias Médicas Volumen 44 número 4 año 2019 


\section{Introducción}

La Comisión sobre Determinantes Sociales, iniciativa de la Organización Mundial de la Salud es enfática al señalizar que, en los países la salud y enfermedad siguen una gradiente donde las personas más pobres tienen el peor estado de salud. Además, recomienda medir la magnitud del problema, analizarlo y evaluar los efectos de las intervenciones realizadas para controlarlo (Comisión sobre los determinantes Sociales de la Salud, 2008).

Existen ejemplos de este fenómeno en América Latina: un estudio colombiano relaciona el riesgo de morir por enfermedad isquémica e hipertensiva según un índice de necesidades básicas insatisfechas, el mayor riesgo se situó entre las municipios más pobres (Cardona et al., 2015). En Perú, se ha observado que las personas no indígenas que viven en la Amazonía peruana en extrema pobreza presentan un OR 2,31 (IC 95\% 1,50 - 3,55) de tener malnutrición crónica (Díaz et al., 2015).

Por otro lado, las intoxicaciones son un problema global de salud pública. Según los datos de la Organización Mundial de la Salud (OMS), se estima un total de 193460 muertes en el mundo por intoxicaciones no intencionales en el año 2012. Además, se reportan cerca de un millón de muertes por suicidio anuales, dentro de las cuales 370000 corresponden a ingestión intencional de plaguicidas (World Health Organization, 2017). El año 2008 la OMS también informó que las intoxicaciones son la quinta causa de mortalidad en la población infantil, con más de 45000 niños muertos al año en el mundo (World Health Organization, 2008). En Chile, el Centro de información toxicológica y de medicamentos de la Pontificia Universidad Católica de Chile (CITUC) en su última memoria del segundo semestre del año 2017 destaca que los casos de exposición en humanos son, en promedio, alrededor de 26000 anuales (Centro de Información Toxicológica y de Medicamentos, 2017).

Existe información de la incidencia de las exposiciones a sustancias tóxicas alrededor del mundo, pero la evidencia que busca explicar las causas o factores condicionantes de las exposiciones a sustancias con potencial tóxico es muy limitada, a pesar de que este tipo de información constituye un insumo importante para elaborar e implementar medidas de prevención y control en salud pública. En este contexto, y para intentar explorar el fenómeno de "exposiciones agudas a sustancias tóxicas y posible relación con los determinantes sociales en salud", se plantean las siguientes hipótesis:

1. Las exposiciones a sustancias tóxicas no se distribuyen de forma aleatoria en la población, por lo tanto, hay factores que la condicionan.
2. La pobreza es un determinante social de la salud y tal como se ha visto con otros resultados en salud, las personas con mayor pobreza concentran la morbilidad, por lo tanto, ocurriría lo mismo con las exposiciones a sustancias tóxicas, existiendo una gradiente que depende de la situación socioeconómica de las personas.

De acuerdo con estas hipótesis se planteó la pregunta de investigación de este estudio: ¿De qué manera el nivel socioeconómico de las personas se asocia a las características de las exposiciones?

Los objetivos de esta investigación fueron:

1. Evaluar si existe alguna relación entre el nivel de pobreza y la exposición aguda a sustancias con potencial tóxico.

2. Identificar variables que están condicionadas por la pobreza de la población.

3. Evaluar la posible magnitud de las relaciones en las variables condicionadas.

\section{Materiales y métodos}

El presente estudio exploratorio es de tipo transversal y semi-ecológico. Se seleccionó una muestra por conveniencia, correspondiente a todos los casos atendidos por uno de los profesionales del CITUC entre el 1 de enero y el 6 de febrero del 2018. Se definió como caso, aquel llamado recibido por el centro en cual hubo una persona expuesta a una sustancia con potencial tóxico.

La exposición o variable independiente corresponde al nivel de pobreza de cada persona, entendida como la condición en la cual las personas no poseen los recursos suficientes para poder cubrir de manera adecuada las necesidades básicas: alimentación, vestuario, vivienda, educación y salud, entre otras (Ministerio de Desarrollo Social, 2011). Para representarla se utilizó el porcentaje de pobreza comunal determinada por ingreso, disponible en la Encuesta de Caracterización Socioeconómica Nacional (CASEN) 2015 (Ministerio de Desarrollo Social, 2015), imputándose el nivel de pobreza comunal a los casos existente de acuerdo al lugar donde se encontraba la persona al momento de ocurrido el hecho, información obtenida por la persona que atendió el caso.

El resultado o variable dependiente corresponde a las características de los casos de las exposiciones a sustancias con potencial tóxico atendidas por CITUC: edad de la persona expuesta, circunstancia de la exposición, agente involucrado, tipo de exposición (por ejemplo: aguda, crónica), sexo, lugar desde donde se efectuó el primer llamado al centro toxicológico y hora de la ocurrencia del llamado al centro toxicológico. 
Los datos se extrajeron desde la ficha física oficial de CITUC que se completa para cada caso, los cuales fueron posteriormente tabulados en Microsoft Excel ${ }^{\circledR}$ (Microsoft Corporation, 2016). Luego, se categorizaron las variables para su descripción y luego se re-categorizaron para poder realizar el análisis estadístico.

Se ejecutó un análisis univariable a través de tablas cruzadas y pruebas de significancia de Chi- Cuadrado con $p<0,05$. Se categorizó la variable "pobreza" formando 2 grupos con corte en la mediana, comparándose el grupo sobre el percentil 50 con el grupo bajo el percentil 50 de pobreza. Las personas en el percentil mayor de pobreza representan a aquellas con un nivel socioeconómico menor. Este análisis se realizó con el programa IBM SPSS ${ }^{\circledR}$ (IBM Corp, 2017).

Luego del análisis univariado, se calcularon Odds Ratios (ORs) y análisis estratificado con las variables que resultaron estadísticamente significativas en el análisis previo, con un valor $\mathrm{p}<0,05$ e intervalos de confianza de $95 \%$, el cual fue realizado en programa OpenEpi ${ }^{\oplus}$ (Dean et al.,2013).

Se excluyeron para los análisis respectivos aquellos casos con datos en blanco y/o los "no clasificables en otras categorías. También se excluyó en este proceso la variable tipo de exposición, por comportarse como una constante.

La investigación fue evaluada y autorizada por el comité ético científico de la Facultad de Medicina UC.

\section{Resultados}

\section{A.Descripción general de la muestra}

La muestra final fue de un total de 397 casos. Los casos incluidos en el estudio corresponden a 133 comunas del país, las cuales presentan índices de pobreza por ingresos que van desde 0,3\% hasta el 40,8\%. Los llamados al CITUC fueron realizados mayoritariamente desde centros asistenciales, los cuales dan cuenta del $58,2 \%$, en segundo lugar, se sitúan los llamados realizados desde el domicilio, con un $34,5 \%$. Un $94,7 \%$ de los casos ocurrieron en los domicilios de los afectados.
En relación con las características de los casos, se observó una mayor proporción de éstos en el sexo femenino, con un 57\% del total. La vía de exposición principal fue la ingestión, con un $82,6 \%$ de los casos, seguido por la vía inhalatoria con un $6,5 \%$. Las circunstancias de la exposición fueron principalmente accidentales con un $50,1 \%$ e intencionales con un $32,0 \%$. Las exposiciones fueron primordialmente agudas, concentrando el $89,4 \%$ de los casos. La distribución de los agentes involucrados en los casos, corresponden en su mayoría a medicamentos, con un 52,9\%, seguido de productos de uso doméstico, con un $22,2 \%$.

\section{B. Análisis univariable}

Respecto del análisis univariable, para relacionar pobreza y exposiciones agudas a sustancias tóxicas, en la tabla 1 se presentan los resultados de las tablas cruzadas y sus respectivas pruebas de significancia con chi-cuadrado. Destaca que únicamente las variables dependientes "circunstancia" y "lugar de llamado" tienen diferencias significativas al ser analizadas bajo la variable independiente "pobreza", observándose una tendencia a que las personas que viven en comunas con un índice de pobreza mayor tienen más exposiciones intencionales y muestran una proporción mayor de consultas desde los centros de salud, en comparación con aquellas personas provenientes de comunas con menor índice de pobreza, donde se observa una mayor proporción de circunstancias accidentales y consultas realizadas desde sus domicilios.

No se encontraron diferencias significativas en las distribuciones por edad, vías de exposición y las horas en la que ocurren las consultas por estas situaciones de acuerdo al nivel de pobreza.

\section{Análisis estratificados}

Se observa un OR ajustado de 2,47 (IC 95\%: 1,47 - 4,12) de posibilidad de que en el grupo de pobreza mayor al p50, el primer llamado al centro toxicológico por exposición provenga de un centro asistencial en comparación con el grupo de pobreza bajo el p50 (tabla 2). 
Medel et al.

Tabla 1: Tabla cruzada entre grupos de variable independiente pobreza sobre y bajo percentil 50, con variables dependientes hora del llamado, edad del expuesto.

*: valor p significativo

\begin{tabular}{|c|c|c|c|c|c|c|}
\hline & \multirow[t]{2}{*}{ Categoría } & \multicolumn{2}{|c|}{ Nivel pobreza bajo P50 } & \multicolumn{2}{|c|}{ Nivel pobreza sobre P50 } & \multirow{2}{*}{$\begin{array}{c}\text { Valor } p \\
(\mathrm{Xi2})\end{array}$} \\
\hline & & \% & $\underline{\mathbf{n}}$ & \% & $\underline{\mathbf{n}}$ & \\
\hline Hora & $\begin{array}{c}\text { 18-24 horas } \\
\text { 12-18 horas } \\
\text { 6-12 horas } \\
\text { 0-6 horas } \\
\text { Total }\end{array}$ & $\begin{array}{c}38,9 \% \\
35,5 \% \\
10,8 \% \\
14,8 \% \\
100,0 \%\end{array}$ & $\begin{array}{l}79 \\
72 \\
22 \\
30 \\
203\end{array}$ & $\begin{array}{c}42,3 \% \\
34,0 \% \\
13,9 \% \\
9,8 \% \\
100,0 \%\end{array}$ & $\begin{array}{l}82 \\
66 \\
27 \\
19 \\
194\end{array}$ & 0,38 \\
\hline Edad & $\begin{array}{l}20 \text { o más años } \\
0 \text { a } 9 \text { años } \\
10 \text { a } 19 \text { años } \\
\text { Total }\end{array}$ & $\begin{array}{l}42,8 \% \\
42,8 \% \\
14,4 \% \\
100,0 \%\end{array}$ & $\begin{array}{l}86 \\
86 \\
29 \\
201\end{array}$ & $\begin{array}{l}40,9 \% \\
39,4 \% \\
19,7 \% \\
100,0 \%\end{array}$ & $\begin{array}{l}79 \\
76 \\
38 \\
193\end{array}$ & 0,38 \\
\hline Sexo & $\begin{array}{l}\text { Femenino } \\
\text { Masculino } \\
\text { Total }\end{array}$ & $\begin{array}{l}54,5 \% \\
45,5 \% \\
100,0 \%\end{array}$ & $\begin{array}{l}110 \\
92 \\
202\end{array}$ & $\begin{array}{l}59,8 \% \\
40,2 \% \\
100,0 \%\end{array}$ & $\begin{array}{l}116 \\
78 \\
194\end{array}$ & 0,28 \\
\hline Lugar de Llamado & $\begin{array}{l}\text { Instituciones de Salud } \\
\text { Domicilio } \\
\text { Total }\end{array}$ & $\begin{array}{l}53,1 \% \\
46,9 \% \\
100,0 \%\end{array}$ & $\begin{array}{l}104 \\
92 \\
196\end{array}$ & $\begin{array}{l}76,6 \% \\
23,4 \% \\
100,0 \%\end{array}$ & $\begin{array}{l}147 \\
45 \\
192\end{array}$ & $<0,05^{*}$ \\
\hline Circunstancia & $\begin{array}{l}\text { Accidental } \\
\text { Intencional } \\
\text { Total }\end{array}$ & $\begin{array}{l}70,3 \% \\
29,7 \% \\
100,0 \%\end{array}$ & $\begin{array}{l}130 \\
55 \\
185\end{array}$ & $\begin{array}{c}54,3 \% \\
45,70 \% \\
100,00 \%\end{array}$ & $\begin{array}{r}94 \\
79 \\
173\end{array}$ & $0,02^{*}$ \\
\hline Mezcla de agentes & $\begin{array}{l}\text { Agente Único } \\
\text { Multiagente } \\
\text { Total }\end{array}$ & $\begin{array}{l}82,8 \% \\
17,2 \% \\
100,0 \%\end{array}$ & $\begin{array}{l}168 \\
35 \\
203\end{array}$ & $\begin{array}{l}78,40 \% \\
21,60 \% \\
100,00 \%\end{array}$ & $\begin{array}{l}152 \\
42 \\
194\end{array}$ & 0,267 \\
\hline Tipo de Agente & $\begin{array}{c}\text { Medicamentos } \\
\text { Agentes de uso doméstico } \\
\text { Agentes de uso NO doméstico } \\
\text { Total }\end{array}$ & $\begin{array}{l}56,8 \% \\
28,4 \% \\
14,8 \% \\
100,0 \%\end{array}$ & $\begin{array}{l}100 \\
50 \\
26 \\
176\end{array}$ & $\begin{array}{l}61,50 \% \\
21,20 \% \\
17,30 \% \\
100,00 \%\end{array}$ & $\begin{array}{l}110 \\
38 \\
31 \\
179\end{array}$ & 0,283 \\
\hline Vía de Exposición & $\begin{array}{c}\text { Ingestión } \\
\text { Cutánea, Ocular, Mordeduras } \\
\text { Picaduras } \\
\text { Inhalación } \\
\text { Total }\end{array}$ & $\begin{array}{l}80,1 \% \\
13,4 \% \\
6,5 \% \\
100,0 \%\end{array}$ & $\begin{array}{l}161 \\
27 \\
13 \\
201\end{array}$ & $\begin{array}{c}86,50 \% \\
6,70 \% \\
6,70 \% \\
100,00 \%\end{array}$ & $\begin{array}{l}167 \\
13 \\
13 \\
193\end{array}$ & 0,89 \\
\hline
\end{tabular}


Medel et al.

Tabla 2: Primer análisis estratificado, resultados crudo para variables pobreza y lugar de llamado.

\begin{tabular}{|c|c|c|c|c|c|c|}
\hline & $\begin{array}{l}\text { Instituciones de } \\
\text { Salud }\end{array}$ & Domicilio & Total & OR crudo & $\begin{array}{c}\text { Breslow Day: } \\
\text { Valor } p\end{array}$ & $\begin{array}{l}\text { OR Ajustado } \\
\text { Mantel Henzel }\end{array}$ \\
\hline Pobreza sobre p50 & 132 & 39 & 171 & \multirow{3}{*}{$\begin{array}{c}2,80 \\
(1,76-4,44)\end{array}$} & \multirow{3}{*}{0,45} & \multirow{3}{*}{$\begin{array}{c}2,47 \\
(1,47-4,12)\end{array}$} \\
\hline Pobreza bajo p50 & 98 & 81 & 179 & & & \\
\hline Total & 230 & 120 & 350 & & & \\
\hline
\end{tabular}

Por otro lado, se observa un OR ajustado de 0,76 (IC 95\%: 0,47 -

las exposiciones sean por circunstancia accidental en comparación 1,25) de posibilidad de que en el grupo de pobreza mayor al p50, con el grupo de pobreza bajo el p50 (Tabla 3).

Tabla 3: Segundo análisis estratificado, resultados crudos para variables pobreza y circunstancias de exposición.

\begin{tabular}{|c|c|c|c|c|c|c|}
\hline & Accidental & Intencional & Total & OR crudo & Breslow Day: Valor p & $\begin{array}{c}\text { OR Ajustado Mantel } \\
\text { Henzel }\end{array}$ \\
\hline Pobreza sobre p50 & 93 & 78 & 171 & \multirow{3}{*}{$\begin{array}{c}0,51 \\
(0,31-0,82)\end{array}$} & \multirow{3}{*}{0,45} & \multirow{3}{*}{$\begin{array}{c}0,76 \\
(0,47-1,25)\end{array}$} \\
\hline Pobreza bajo p50 & 125 & 54 & 179 & & & \\
\hline Total & 218 & 132 & 350 & & & \\
\hline
\end{tabular}

\section{Discusión}

De acuerdo con los resultados de esta investigación exploratoria, los determinantes sociales de la salud influyen en la presentación de las exposiciones a sustancias tóxicas en Chile, sustentando la hipótesis que da origen a este trabajo.

Se observan diferencias significativas en relación con las circunstancias, o, en otras palabras, del porqué de las exposiciones. De acuerdo con los resultados, las personas que viven en comunas con mayor porcentaje de pobreza tendrían más exposiciones intencionales en comparación a aquellas personas de comunas con menor porcentaje de pobreza (45,70\% versus $29,70 \%)$. Se puede especular que las necesidades básicas no cubiertas en las personas que viven en comunas con niveles de mayor pobreza pueden propiciar un aumento de las exposiciones intencionales, dentro de las que se sitúan los intentos suicidas. Este antecedente puede ser muy relevante para el área de salud mental.

En segundo lugar, destaca el hecho de que no se encontraron diferencias significativas en las distribuciones por edad, vías de exposición y las horas en la que ocurren las consultas por estas situaciones de acuerdo con el nivel de pobreza. Esta observación puede ser evidencia de que existiría un patrón común del cómo ocurren las exposiciones de la población en el país, independiente de su situación socioeconómica.

Que se sustente la relación entre"exposiciones agudas a sustancias tóxicas y posibles condicionantes" es importante, ya que podría abrir una línea investigativa que puede generar medidas de control de las intoxicaciones en el mediano o largo plazo. Por ejemplo, se podrían diseñar intervenciones en salud, especialmente desde el punto de vista de la salud mental para disminuir la frecuencia y consecuencias de exposiciones intencionales en los grupos con mayor proporción de pobreza en el país, con foco en la prevención de intentos suicidas.

Por otro lado, un hallazgo tangencial que puede ser interesante es el relacionado con el distinto uso que da la población al sistema de salud dependiendo de su situación socioeconómica. Se observó que las personas de comunas con mayor nivel de pobreza tienen la tendencia a consultar primero en centros asistenciales antes que efectuar telefónicamente la consulta a un Centro toxicológico, donde el primer grupo concentró el 76,60\% de los Ilamados desde centros asistenciales de salud, versus un 53,10\% en el segundo grupo. Esto es de alto interés para políticas de salud pública, porque existe una proporción importante de personas que consultan en los centros asistenciales por exposición a sustancias potencialmente tóxicas y que, por su bajo riesgo, no requieren evaluación presencial por un profesional del equipo de salud y/o que podrían ser manejados en casa con cuidados básicos recomendados telefónicamente por el centro toxicológico.

Bajo este contexto, si en Chile todas las personas expuestas a alguna sustancia con potencial tóxico consultaran en un centro toxicológico antes de presentarse en un servicio de urgencia, se podría descongestionar a estos últimos y ahorrar dinero al sistema. 
Esta idea es apoyada en la literatura científica, donde en estudios realizados en Singapur y Estados Unidos reportan ahorros entre 110000 y 600000 pesos chilenos en promedio por cada paciente que es atendido por un centro toxicológico y que no llega al servicio de urgencia (Miller \& Lestina, 1997; Ponampalam \& Loh, 2010). Tomando en consideración el ahorro por paciente y el volumen de pacientes que se expone de forma aguda a sustancias tóxicas cada año y que consultan en el CITUC, el ahorro que puede generar el centro toxicológico al sistema de salud puede ser considerable.

Respecto a las causas de esta situación que es interesante, lamentablemente no se puede decir mucho por el diseño ecológico de esta investigación, sin embargo, es muy posible que tenga relación con un fenómeno de mayor envergadura, que es el de la congestión de los servicios de urgencia que ocurre en Chile (Salway, et al., 2017).

En cuanto a los métodos del estudio, sus principales fortalezas tienen relación con la calidad de los datos utilizados, los cuales son tomados de forma sistemática y en una institución que es referente nacional en toxicología en el país. Además, el registro de los datos fue realizado por la misma persona, lo que implica uniformidad de la recolección. La muestra obtenida, el número de casos analizados y el cómo se distribuyeron las variables permitió hacer un análisis en todas las áreas de interés y algunas pruebas de significancia.

Respecto a las posibles debilidades y sesgos del estudio, hay un punto crítico a considerar en su interpretación: la imputación del nivel de pobreza de acuerdo con la comuna reportada del caso. Sabemos que el índice comunal de pobreza no es un estimador inequívoco de las condiciones socioeconómicas de todas las personas que viven en la comuna, ya que en comunas con altos niveles de pobreza existen personas con altos ingresos, y viceversa. Por lo tanto, la metodología no permite diferenciar entre estas personas, y las clasifica a todas según el nivel de pobreza comunal. Además, no se consideraron otras variables que podrían tener un impacto en el fenómeno de estudio, de allí su naturaleza exploratoria. Por ello, existe una fuente de sesgo que se debe considerar al momento de hacer la interpretación de los resultados presentados. Otra debilidad es que se tomo una muestra de los casos atendidos por el Centro, ya que se analizaron 397 de más de 25000 que se atienden al año. Esto fue por razones prácticas, ya que en ese momento, el sistema de registro electrónico tenía deficiencias en el registro de variables de interés para esta investigación, por lo tanto, se optó por tener un menor número de casos, con una mayor calidad en el registro de los datos. Sin embargo, si este número reducido logra detectar diferencias en la exposición a sustancias tóxicas de acuerdo al estado socioeconómico, se puede especular que otro estudio con un número mayor de sujetos va a mantener la tendencia, y reflejar mejor la magnitud de dichas diferencias.
Sin embargo, considerando la naturaleza exploratoria de este proyecto, se justifica haber realizado un diseño de investigación con características semi-ecológica, para tener un primer acercamiento al fenómeno y poder plantear nuevas preguntas de investigación.

De acuerdo con lo anterior, el estudio cumple con el objetivo de explorar la relación pobreza-intoxicaciones, ya discutida en las secciones anteriores, observándose la existencia una relación entre la pobreza (un fenómeno de carácter social) y las exposiciones a sustancias tóxicas.

Por último, de este estudio exploratorio nacen los nuevos desafíos que podrían cimentar una línea de investigación. Es necesario comenzar con investigaciones que busquen dar respuestas a las nuevas preguntas planteadas, como por ejemplo: ¿Existe una concentración en la distribución de las exposiciones más graves de acuerdo a variables socioeconómicas?; ¿Los centros de salud están preparados para atender los casos de intoxicaciones?, ¿Cómo se está manejando las intoxicaciones junto al componente psiquiátrico en los intentos suicidas?, cuyas respuestas aportarían a conocer con mayor detalle qué medidas deben tomarse para cómo disminuir la incidencia de las intoxicaciones agudas y manejar a los pacientes de forma óptima.

\section{Agradecimientos}

Se agradece a CITUC por permitir usar sus datos para la investigación, al Magister en salud pública de la Pontificia Universidad Católica de Chile, programa en el cual se desarrolló la tesis que da origen a este artículo.

\section{Referencias}

Cardona D, Cerezo M del P, Parra H, Quintero L, Muñoz L, Cifuentes OL, \& Vélez SC. (2015). Desigualdades en la mortalidad por enfermedades cardiovasculares en los municipios del Eje Cafetero, 2009-2011. Biomédica, 35, 379-94.

Centro de Información Toxicológica y de Medicamentos. (2017). MEMORIA 25 AÑOS CITUC. Santiago de Chile. Accedido en: http:// cituc.uc.cl/cituc-memoria/ el 05 de junio de 2019.

Comisión sobre los determinantes Sociales de la Salud. (2008). Subsanar las desigualdades en una generación. Global Health Promotion, 40.

Dean, A., Sullivan, K., \& Soe, M. (2013). OpenEpi: Open Source Epidemiologic Statistics for Public Health. Accedido en: www. OpenEpi.com el 07 de julio de 2019. 


\section{Medel et al.}

Díaz A, Arana A, Vargas-machuca R. \& Antiporta, D. (2015). Situación de salud y nutrición de niños indígenas y niños no indígenas de la Amazonia peruana. Revista Panamericana de Salud Pública 38,49-56.

IBM Corp. (2017). IBM SPSS Statistics for Windows, Version 25. Armonk, NY.

Medel-Jara PA. Lopez Nuñez A, Ramirez M, Silva L \& Ríos JC. (2018). ¿La pobreza es un factor de riesgo para las intoxicaciones en Chile? Jornadas SoTox 2018. Valparaíso, Chile.

Microsoft Corporation. (2016). Microsoft Excel. Redmond, Washington.

Miller TR \& Lestina DC. (1997). Costs of Poisoning in the United States and Savings From Poison Control Centers: A Benefit- Cost Analysis. Annals of Emergency Medicine 29, 239-245.

Ministerio de Desarrollo Social. (2015). Serie de Datos de la Tasa de Pobreza Comunal. Accedido en: http://observatorio.ministeriodesarrollosocial.gob.cl/indicadores/datos_pobreza_comunal.php el 12 de noviembre de 2019.
Ministerio de Desarrollo Social. (2011). Informe de Politica Social. Accedido en: http://www.desarrollosocialyfamilia.gob.cl/storage/ docs/ipos_2011.pdf el 10 de octubre de 2019.

Ponampalam R \& Loh CS. (2010). Cost benefits of the Drug and Poison Information Centre in preventing unnecessary hospitalisation: The Singapore experience. Hong Kong Journal of Emergency Medicine 17, 45-53.

Salway R, Valenzuela R, Shoenberger J, Mallon W. \& Viccellio A. (2017). Congestión En El Servicio De Urgencia: Respuestas Basadas En Evidencias a Preguntas Frecuentes. Revista Médica Clínica Las Condes 28, 220-227.

World Health Organization. (2008). Cada día mueren más de 2000 niños por lesiones no intencionales. Accedido en: https://www. who.int/mediacentre/news/releases/2008/pr46/es/ el 15 de noviembre de 2019.

World Health Organization. (2017). Poisoning Prevention and Management. Accedido en: https://www.who.int/ipcs/poisons/ en/ el 14 de noviembre de 2019. 